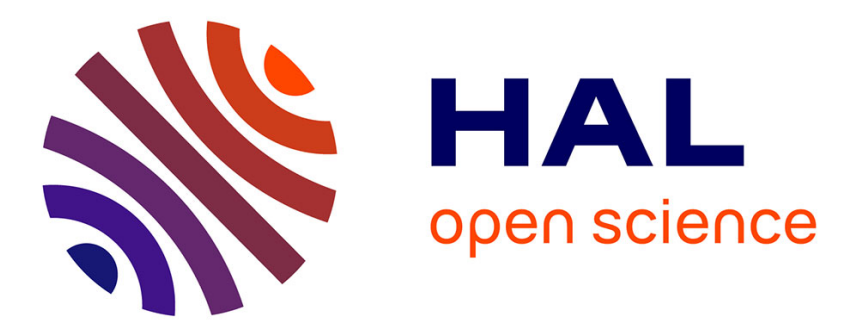

\title{
CIRCULARLY POLARIZED ANTENNA ON SOI FOR THE 60 GHZ BAND
}

Moussa Barakat, Christophe Delaveaud, Fabien Ndagijimana

\section{To cite this version:}

Moussa Barakat, Christophe Delaveaud, Fabien Ndagijimana. CIRCULARLY POLARIZED ANTENNA ON SOI FOR THE 60 GHZ BAND. The Second European Conference on Antennas and Propagation (EuCAP 2007), Nov 2007, Edinburgh, United Kingdom. hal-00194662

\section{HAL Id: hal-00194662 \\ https://hal.science/hal-00194662}

Submitted on 7 Dec 2007

HAL is a multi-disciplinary open access archive for the deposit and dissemination of scientific research documents, whether they are published or not. The documents may come from teaching and research institutions in France or abroad, or from public or private research centers.
L'archive ouverte pluridisciplinaire HAL, est destinée au dépôt et à la diffusion de documents scientifiques de niveau recherche, publiés ou non, émanant des établissements d'enseignement et de recherche français ou étrangers, des laboratoires publics ou privés. 


\title{
CIRCULARLY POLARIZED ANTENNA ON SOI FOR THE 60 GHZ BAND
}

\author{
M. Barakat ${ }^{*}$, C. Delaveaud $*$ F. Ndagijimana ${ }^{\dagger}$ \\ * Commissariat à l'énergie atomique (CEA/LETI, MINATEC) \\ Moussa.barakat@cea.fr; christophe.delaveaud@cea.fr \\ †Institut de Microelectronics, Electromagnetisme et Photonique (IMEP) \\ Fabien.ndagijimana@minatec.inpg.fr \\ Grenoble, France
}

Keywords: $60 \mathrm{GHz}$, Spiral antenna, Coplanar strip,
integrated antenna, Silicon on insulator

\begin{abstract}
The performance of a dual-arm millimeter wave Archimedean spiral antenna integrated on SOI is described. The impact of the feeding structure on the return loss and the axial ratio is displayed. A good return loss with $35 \mathrm{GHz}-10 \mathrm{~dB}$ bandwidth and good circular polarization over $120^{\circ}$ elevation angle independent on the azimuth angle (less than $-5 \mathrm{~dB}$ axial ratio) are obtained. The simulated efficiency is $80 \%$ with a gain of $4.3 \mathrm{dBi}$.
\end{abstract}

\section{Introduction}

$\mathbf{W}$ ith the current trend of wireless technology innovation there is a vast array of wireless applications, ranging from space to shipboard applications, which require antennas that are low cost, compact, and broadband. Spiral antennas have characteristics that are essential to wireless communications in many applications. Their wide-band characteristics combined with their low profile as well as their low cost make these antennas prime candidates for use in sensors and some mobile applications [5].

The utilization of millimeter wave frequencies enables the design of compact, lightweight, and low cost wireless millimeter wave communication front-ends which can offer convenient terminal mobility and high capacity channel. The $60 \mathrm{GHz}$ band is attractive candidate for short range radar and indoor communications based on Pico-cell zone because it presents high atmospheric loss which limits interference between cells. The design of integrated antennas operating at $60 \mathrm{GHz}$ is a critical issue when high efficiency is required [3]. Recently, Silicon on Insulator (SOI) technology was found to offer alternative solutions to many problems faced in the race to higher performance and low power of integrated devices [7].

At the same time, SOI does not require major changes compared to the well known complementary metal oxide semiconductor (CMOS) process flow. The performance of traditional microwave circuits such as transmission lines and antennas, on low resistivity silicon wafer, is problematic due to the high losses for example on CMOS substrate [7]. In most literature dealing with circularly polarized antennas, axial ratio is taken as one of the primary criteria for evaluating the quality of the antenna; Improper feed, higher order band radiation or a combination of the two will contribute to this effect [4].

Traditional implementation of Archimedean spiral antennas entails using a perfect electric conducting (PEC) ground plane placed at a distance of $\lambda_{g} / 4$ below the antenna to produce a unidirectional beam. This approach introduces a fixed physical length between the antenna and the ground plane in terms of $\lambda_{g}$, thus, seriously limiting the frequency independent characteristics of the antenna [5].

In this article, we present an implementation of an Archimedean spiral antenna integrated on SOI. In the first part, we describe the spiral antenna theory in order to have good radiation properties. Next, the spiral antenna parameters are described; the effect of geometry parameters is studied when integrated on SOI. After the antenna is proposed, the specific feeding structure is showed. The feeding structure is composed of a transition between radiofrequency coplanar waveguide feed, coplanar strip transition and an Archimedean coplanar strip structure. Finally, the simulated result of the return loss, the axial ratio, and the gain diagram are presented.

\section{Spiral Antenna Theory}

Of the variety of possible spiral configurations, only three have received wide attention: the logarithmic or equiangular spiral, the Archimedean or arithmetic spiral, and the rectangular counterpart of the Archimedean spiral. The radiating element may consist of either one or two conductors to form a single or double spiral. The dual-arm spiral has better characteristics than the single arm antenna. As the operating frequency is increased, the physical size of the dual arm antenna becomes so small that it is more difficult to obtain good performance of the antenna [4]

When excited from a balanced two-wire transmission line, these configurations exhibit broadband characteristic with respect to input impedance, radiation pattern, and circular polarization of the radiated field. Since the dual-arm Archimedean spiral possesses the greatest symmetry, only this form is discussed; 
For the determination of an external feeding structure for the spiral antenna, the current distribution and the radiation mechanism of the center-fed spiral have to be carefully considered [9]. From band theory [14] the proper phasing of the feed lines can be derived. The band theory states that the radiation of an Archimedean spiral occurs when currents in neighboring arms of the spiral are in phase. The currents between neighboring arms are in phase after traveling from the center over a certain arm length. Figure 1 shows that currents on opposite arms, shown by arrows as current vectors, are spatially in phase because the arms in the center which extend in opposite directions are fed in antiphase. Therefore currents at position A and position B are in phase. The current at position B' is in phase with the current at $\mathrm{B}$ if the arm length between $\mathrm{A}$ and $\mathrm{B}^{\prime}$ is half a wavelength. Therefore radiation occurs at a circumference of one wavelength [10]. By extending this reasoning, it follows that there is a band of finite width having a mean diameter of $\lambda_{g} / \pi$ in which the current elements in adjacent conductor are in phase, or nearly so.

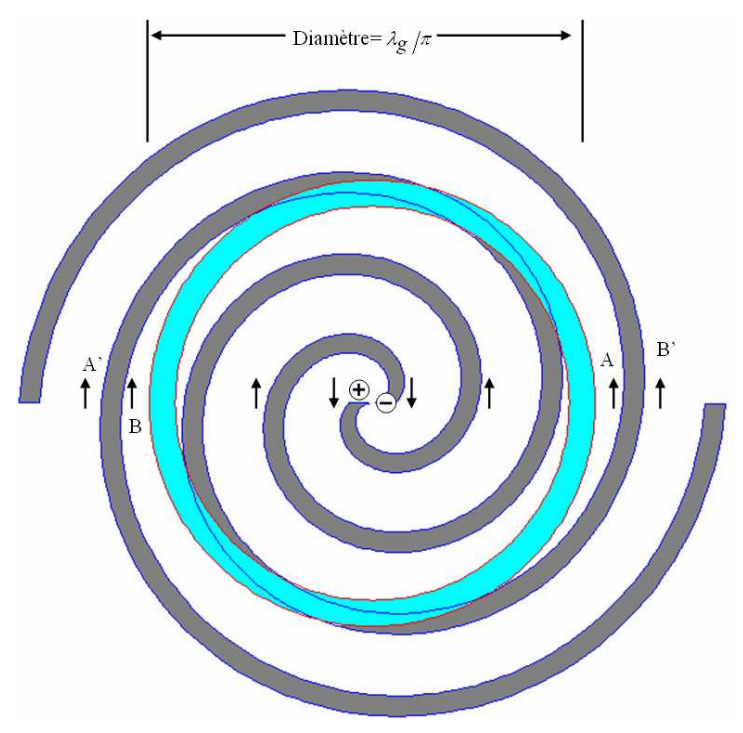

Figure 1: Current distribution in the spiral antenna

This is the normal or fundamental mode and is the one in which nearly all spiral antennas are designed to operate.

To each group of differential current elements lying within the radiating band and on a diameter, there exists a corresponding group which is in time and space quadrature to the first; therefore, the radiation is circularly polarized [4] .

For the second radiation band, this mode of radiation will be dominant when the input terminals of the spiral are excited in phase. For this case, efficient radiation will first occur from a band whose mean circumference is $2 \lambda_{g} / \pi$. In contrast to the previous case, differential current elements diametrically opposite are out of phase since the elements are now separated by one wavelength. Moreover, the primed and unprimed current elements are out of phase, hence the radiation pattern has a null along the axis normal to the plane of the spiral.

\section{Antenna Parameters}

Each arm of an Archimedean spiral is linearly proportional to the angle, $\theta$ and is described by the following relationships

$$
r=r_{0} \theta+r_{1}
$$

And

$$
r=r_{0}\left(\theta+\theta_{0}\right)+r_{1}
$$

Where

$r$ and $\theta$ are conventional polar coordinates

$r_{0}, \theta_{0}$ and $r_{1}$ are constants.

The width of the spiral arm $W$ is determined by multiplying $r_{0}$ and $\theta_{0}$. The effect of this factor will be studied based on the return loss and the axial ratio.

In general the second conductor is generated by rotating the first conductor 180 degrees about the origin.

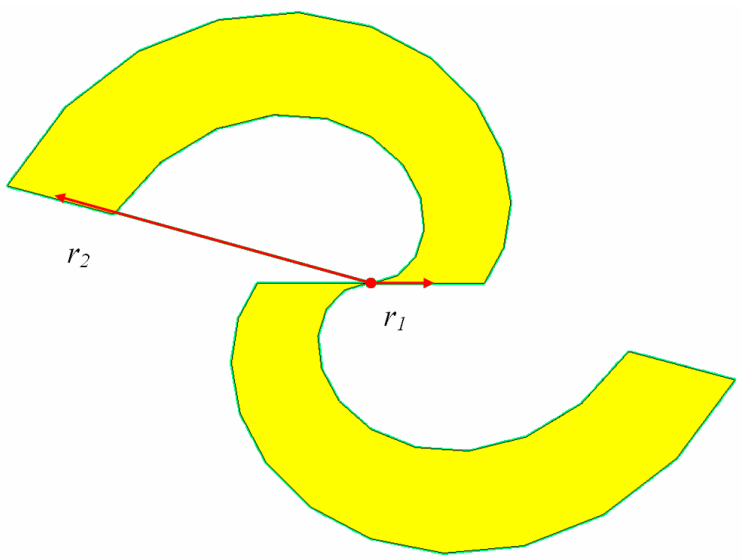

Figure 2 : Spiral antenna without feeding

The low frequency operating point of the spiral is determined theoretically by the outer radius $r_{2}$ (Figure 2) and is given by [6]

$$
f_{\text {Low }}=\frac{c}{2 \pi r_{2} \sqrt{\varepsilon_{\text {reff }}}}
$$

Where $c$ is the speed of light.

$\varepsilon_{\text {reff }}$ is the effective permittivity of the SOI substrate

Similarly the high frequency operating point is based on the inner radius $r_{1}$ giving

$$
f_{\text {High }}=\frac{c}{2 \pi r_{1} \sqrt{\varepsilon_{\text {reff }}}}
$$

The input impedance of the antenna is decreased due to SOI substrate's effect. To overcome this problem, the antenna is etched on the 6 copper layers of SOI, connected by metallic vias, and the Alucap layer. These parameters are optimized to 
have a good input impedance matching to $50 \mathrm{ohms}$ including substrate effect. As it has been mentioned before, the lower and upper radii of the spiral antenna control the operating bandwidth. The width of the spiral arm is optimized in order to have a quasi $-10 \mathrm{~dB}$ matching bandwidth. Figure 3 shows the simulated return loss of the dual arm spiral Archimedean antenna. Even tough we use the six layers (M1-M6) and the Alucap layer for the integration of the antenna on SOI (thickness of $\sim 6 \mu \mathrm{m}$ ), the input impedance of the antenna is low, and consequently the return loss is lower than $7 \mathrm{~dB}$ in the operating bandwidth for the $50 \mu \mathrm{m}$ width. By increasing the arm width, we managed to optimize the return loss and improve the matching.

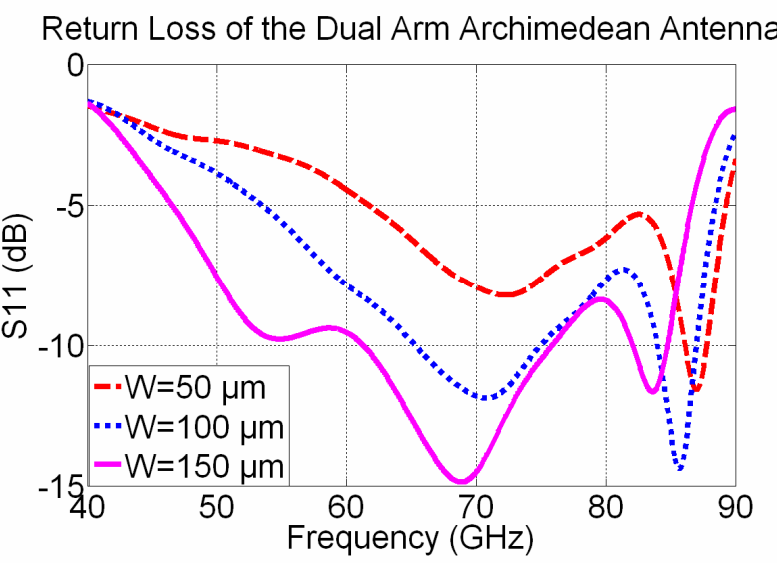

\section{Figure 3 : Return loss of the dual arm Archimedean antenna: Sweep of arm's width}

The second critical parameter, carefully studied during the antenna design, is the axial ratio which determines the circular polarization. An axial ratio of 0 is perfect; a $-5 \mathrm{~dB}$ axial ratio will be acceptable depending on applications. By changing the width of the spiral's arm, the axial ratio is studied. Figure 4 shows the axial ratio of different width of the spiral antenna, the same sweep of the width is used when studying the return loss shown in Figure 3 at $60 \mathrm{GHz}$. We can observe that the axial ratio is: lower than $5 \mathrm{~dB}$ for the three types of width, quasi constant for a $120^{\circ}$ elevation angle.

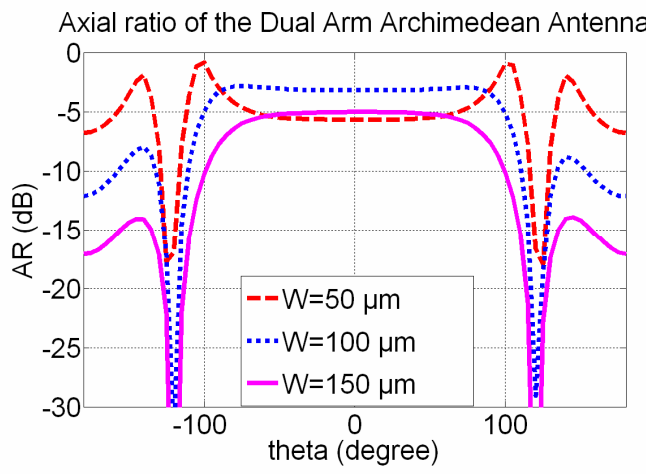

Figure 4 : Axial ratio of the dual arm Archimedean antenna: Sweep of arm's width

\section{Spiral Antenna Feeding}

Feeding system is one of the main problems that the spiral antenna encountered. The entire operation of the spiral antenna in its normal mode was postulated on the basis of an ideal, balanced, two-wire feed structure. Different feeding structure was proposed in the literature; [9] used external feed with integrated microstrip balun to overcome the problem of center feeding; [10] used an integrated balun circuit on a vertical substrate to assure feeding at the spiral centre; [1] used a cavity backed with coaxial feed vertically positioned under the spiral plan to assure balanced feed; Finally [16] used a radio frequency balun with integrated on substrate but positioned on the top of the spiral antenna. We note that, the feeding structure (Figure 5) is sometime as large as the antenna, so that its design will maybe affect the radiation antenna properties and especially the axial ratio.

In our case, we take advantage from the balun design in [16], in order to implement the integrated balun as a first block of our feeding circuit.

The feeding circuit is composed of:

1. A radiofrequency pad, which is used to be the interface between the probe and the antenna for on wafer measurement. The pad has a $100 \mu \mathrm{m}$ pitch and is integrated on the M6 and the Alucap layers of SOI

2. A CPW to CPS transition circuit, which is designed to transform the unbalanced CPW feed line to a balanced CPS (balun) feed line in order to optimize the input of the balanced antenna.

3. A coplanar strip transmission line designed on SOI, the TL is used as an interface between the balun and the spiral coplanar strips

4. A spiral coplanar strip is forming the antiphase feed of the dual arm spiral Archimedean.

The balun is composed of CPS \& CPW TLs separated by a radial slot in the terminated ground, and 2 underpaths connecting the 2 ground planes. Our objective is to approach to the $50 \mathrm{ohms}$ input feed of the designed antenna. The characteristic impedance can be calculated basing on the conformal mapping method by using the formulas (5) [15]

$$
Z_{0, C P W}=\frac{30 \pi}{\sqrt{\varepsilon_{\text {eff }}}} \frac{K(k)}{K\left(k^{\prime}\right)} Z_{0, C P S}=\frac{120 \pi}{\sqrt{\varepsilon_{\text {eff }}}} \frac{K(k)}{K\left(k^{\prime}\right)}
$$

Where

- $Z_{0, C P W}, Z_{0, C P S}$, are the characteristic impedance of the $C P W \& C P S T L$

- $\quad K$ is the elliptic integral of $k$ and $k^{\prime}$ factor that depend on the dimensions of $C P W \& C P S$ (gap and central strip width)

- $\quad \varepsilon_{\text {eff }}$ is the effective permittivity of SOI

The CPS structure is cautiously designed to assure proper feeding, in terms of impedance matching, and in term of geometry. In fact, if we use the same CPS with the same parameters (width of strips and gap), but with a transverse 
configuration (the CPS line is chosen to be perpendicular to the proposed CPS showed in Figure 5, i.e. in the Y direction), the return loss is changed, and the axial ratio is badly damaged. This position is chosen after several optimisations, we have used the feed under the spiral's arm integrated on the first layer of SOI (M1); the result is the presence of different resonance, a modification of the return loss and huge perturbation of the axial ratio radiation pattern. Based on this study, we have chosen to feed the antenna by a spiral coplanar strip, placed inside the dual arm antenna. This configuration was chosen in order to limit radiating alterations. In fact, the energy in the odd excitation will radiate at a diameter of $\lambda_{g} / \pi$. The diameter of the coplanar strip is chosen to be less than $\lambda_{g} / \pi$, so that the phase of the current will not change, and do not obtain large parasitic radiation that will oppose the first radiation mode of the spiral antenna and consequently deteriorate the axial ratio.

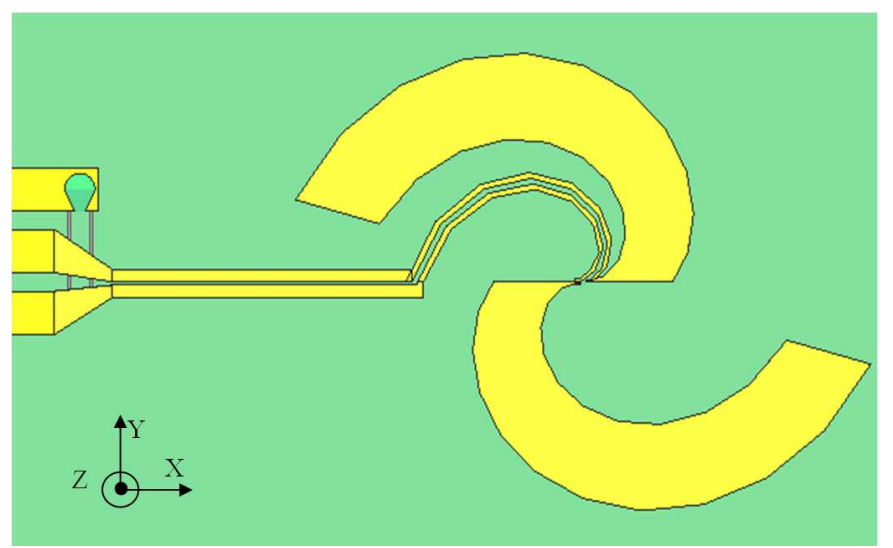

Figure 5 : Schematic of the dual arm Archimedean antenna with feeding structure

The next step for the evaluation of the spiral antenna is studying the effect of the feeding system incorporated at the input of the antenna. Figure 6 shows the return loss of the spiral antenna with the feeding system for different widths. A good matching is found for the spiral with 50 and $100 \mu \mathrm{m}$ widths. The spiral with $150 \mu \mathrm{m}$ width exhibits better return loss with $-15 \mathrm{~dB}$ at $60 \mathrm{GHz}$.

Return Loss of the Dual Arm Archimedean Antenna

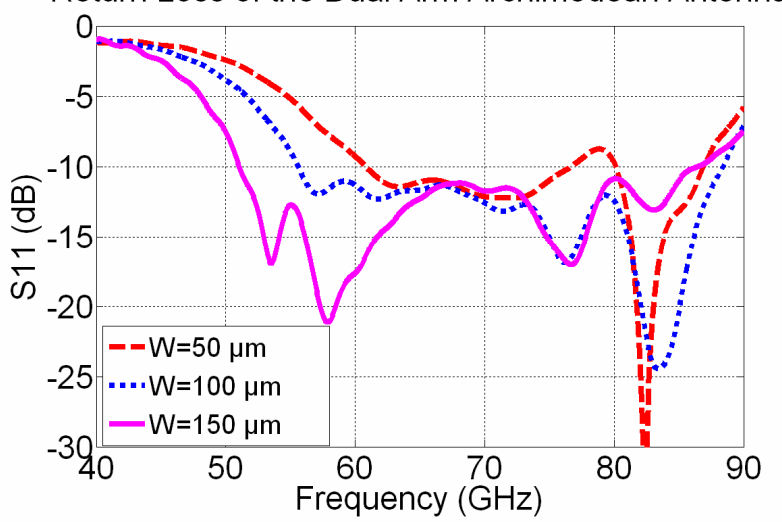

Figure 6 : Return loss of the dual arm Archimedean antenna incorporating the feeding system
In the other hand, the axial ratio is not the same for the different spiral widths. Figure 8 shows the worsening of the antenna axial ratio at $60 \mathrm{GHz}$. We believe that by decreasing the spiral arm, the lower limit of the operating bandwidth is shifting up. This effect will change the axial ratio properties. At $75 \mathrm{GHz}$, the axial ratio was acceptable for the antenna with $50 \mu \mathrm{m}$ arm's width. That is why we have chosen the $150 \mu \mathrm{m}$ width for the design of our antenna.

In addition, the performance of the axial ratio of the $150 \mu \mathrm{m}$ width spiral arm antenna is constant from 55 to $65 \mathrm{GHz}$. Figure 7 shows that we obtain less than $-5 \mathrm{~dB}$ axial ratio for the different frequencies.

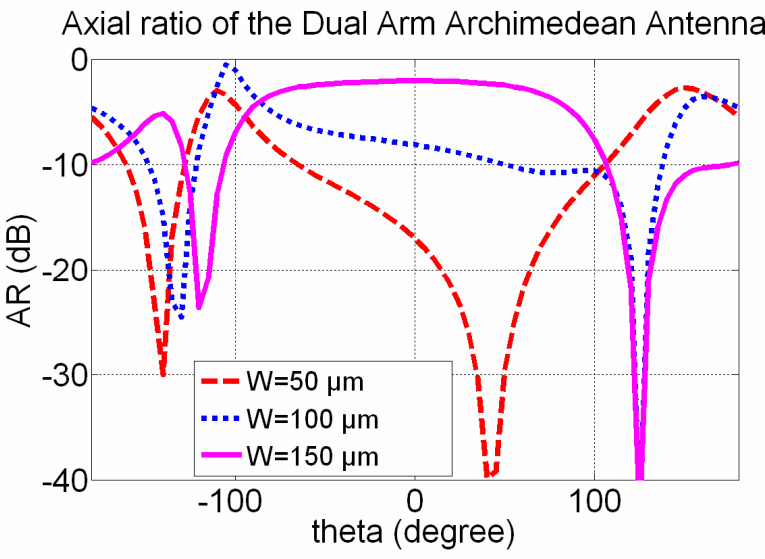

Figure 8 : Axial ratio of the dual arm Archimedean antenna with the feeding structure

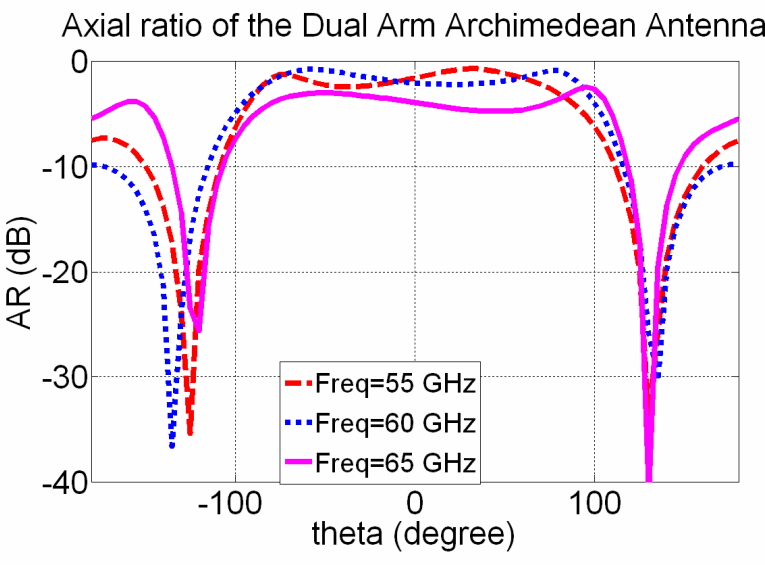

Figure 7 : Axial ratio of the dual arm Archimedean antenna with the feeding structure

\section{Technology Used for Integration}

Dielectric loading offers good possibility for reducing the physical size of an antenna. By increasing the permittivity, the diameter needed, to have first mode radiation of the spiral antenna, is reduced. For example, the CMOS bulk substrate which includes a multi layer of metal, buried oxide layers, and a low resistivity silicon substrate present a relative dielectric permittivity of 11.9 . CMOS bulk reduces 
the size of resonant structure, but the problem with this type of substrate is the high losses. CMOS generate high losses which deteriorate the efficiency of the antenna and thus its gain. However, the recent introduction of high resistive substrate (SOI) reduces the dielectric losses, which prove the importance of SOI for the realization of low loss TL [13]. The topology of SOI substrate is as follows, a high resistive silicon layer ( $>1000$ ohm.cm), six metals layers, buried oxide layers and finally a passivation layer. When not used, the metals layers are replaced by silica.

By using SOI, some design rules are imposed so that the Design Rules Check (DRC) is validated. These rules are:

1. In the SOI technology, an active silicon layer of low resistivity is used to assure transition operation. When it is not used, this layer is replaced by active dummies [8].

2. The second rule is that we have to respect a maximum width of metallic structure. To respect this condition, holes are created inside the antenna's arm.

3. The third rule is the metal density limit. SOI technology imposes that the density of metals in a layer must be between $20 \%$ and $80 \%$ with respect to the overall substrate area [12]. To fulfil this condition, the design of passives dummies is required.

We have studied the effect of dummies by using an adaptation of the Tretyakov models [17] for capacitive surface; we believe that the dummies used in the SOI technology will not affect the performance of the antenna at the $60 \mathrm{GHz}$ frequency band. At their size for the SOI process and when a backside metallization is used, we expect that these dummies will begin to function, at higher frequency ( $>85 \mathrm{GHz})$, as a high impedance surface and may affect the return loss and the radiation pattern.

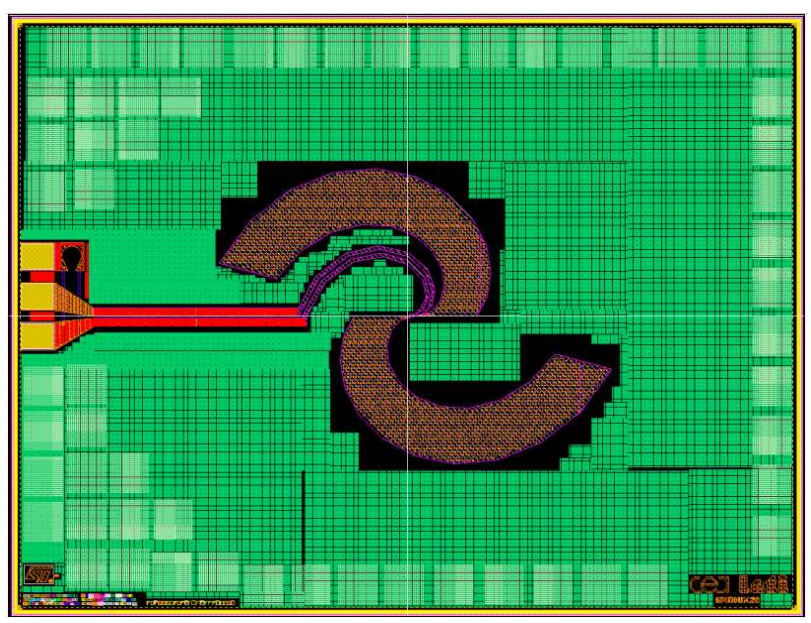

Figure 9 : Schematic of the dual arm Archimedean antenna with feeding structure and dummies
The schematic of the spiral antenna with the dummies is illustrated in Figure 9. The antenna has been drawn using Cadence in order to be compatible with the design rules. We can see in green the dummies used in the antenna.

\section{Radiation}

Classical planar spiral antennas radiate in two broad lobes whose directions are perpendicular to the plane of the antenna. However, a unidirectional pattern is preferred for our application. A backed cavity can be used to redirect the radiation. We have preferred to use a backside metallization, which will be use as a reflector for the radiation. In the same time, this metallization reduces line-to-line coupling and eliminates the parasitic $\mathrm{TE}_{0}$ dielectric slab waveguide mode for the CPS. The distance between radiating arms and the reflector $\left(355 \mu \mathrm{m} \sim \lambda_{g} / 4\right)$ is close to the optimal distance in order to add direct and reflected wave without any side lobes [2][11]. The simulated radiation efficiency is increased (82\% with a gain around $4.2 \mathrm{dBi}$ (Figure 10) compared to spiral configuration without metallization.

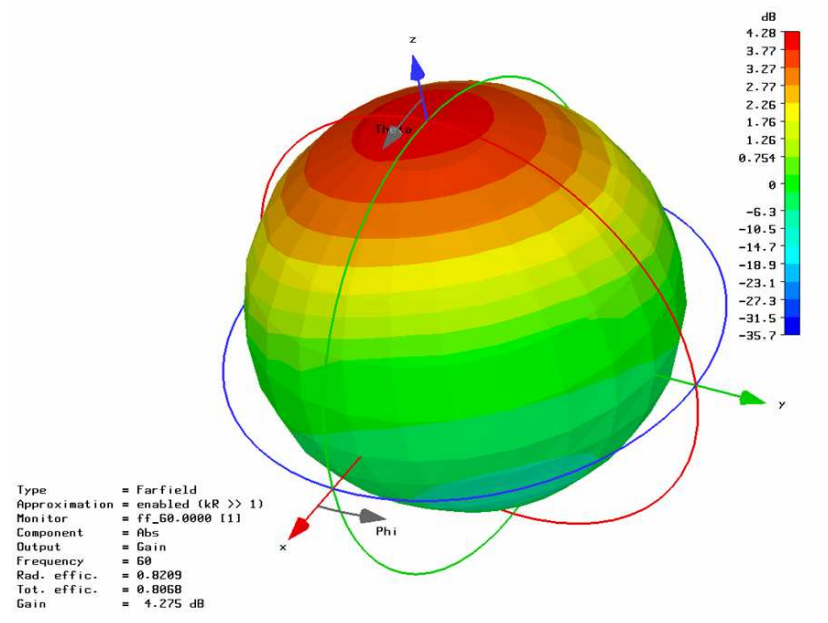

Figure 10 : 3D gain pattern of the spiral antenna.

Finally, the $3 \mathrm{D}$ axial ratio radiation pattern is illustrated in Figure 11. The results reveal a good circular polarization over $120^{\circ}$ elevation angle independent on the azimuth angle.

\section{Conclusion}

This paper has presented the design of a dual arm spiral Archimedean antenna integrated on SOI for the $60 \mathrm{GHz}$ band. At $60 \mathrm{GHz}$ the antenna exhibits a $15 \mathrm{~dB}$ return loss width a $-10 \mathrm{~dB}$ bandwidth of $35 \mathrm{GHz}$. A good circular polarization with an axial ratio lower than $5 \mathrm{~dB}$ was founded. The back side metallization under the wafer is used to act as a reflector. A radiation efficiency of $82 \%$ and a gain around 4.2 $\mathrm{dBi}$ are obtained. This antenna is a part of on chip design using CMOS SOI technology in order to be used in the co- 
design process of the antenna with a $60 \mathrm{GHz}$ integrated Low Noise Amplifier.

The antenna is under realization; we expect to have the antenna and to present measurement of the spiral performance at the time of the conference

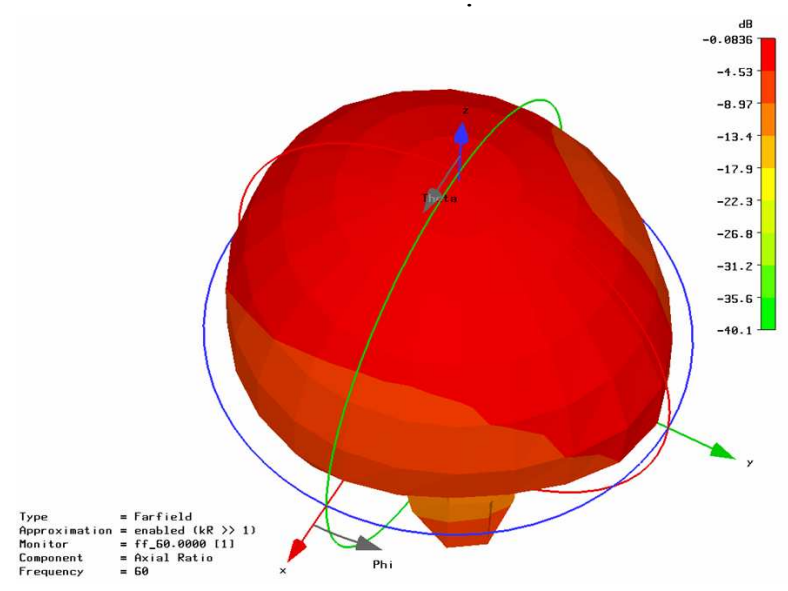

Figure 11:3D patterns for the axial ratio of the spiral antenna.

\section{References}

[1]. M.N. Afsar et al. "A new wideband cavitybacked spiral antenna" 2001 IEEE Antennas and Propagation Society International Symposium, Volume: 4, On page(s): 124-127 vol.4, July 2001

[2]. C. A. Balanis, "Antenna theory analysis and design", p. 453, second edition, John Wiley and Sons, Inc, 1997

[3]. M. H. Barakat et al, "Performance of a $0.13 \mu \mathrm{m}$ SOI integrated $60 \mathrm{GHz}$ dipole antenna", 2007 IEEE Antenna \& Propagation Symposium, Hawaii CDROM June 2007.

[4]. R. Bawer et al., "The spiral Antenna ", IRE International Convention Record, PI. T, pp. 84-95, May, 1960

[5]. J.M. Bell. et al. "A low-profile Archimedean spiral antenna using an EBG ground plane", Antennas and Wireless Propagation Letters, Volume 3, Issue 1, Page(s):223 - 226 (2004)

[6]. Caswell E. D., "Design and Analysis of Star Spiral with Application to Wideband Arrays with Variable Element Sizes", PHD thesis of the Virginia Polytechnic Institute and State University, December 2001

[7]. D. Corson, et al, "Why all the buzz about SOI?" www.rfdesign.com.

[8]. Y. Fukuda et al, "SOI-CMOS Device Technology", OKI Technical review, Number 4, Volume 68, March 2001

[9]. E. Gschwendtner et al. "Spiral antenna with external feeding for planar applications", The 1999 IEEE
AFRICON, Volume 2, and 28 Sept.-1 Oct. 1999 Page: 1011 - 1014 vol.2

[10]. E. Gschwendtner, et al. "Low-cost spiral antenna with dual-mode radiation pattern for integrated radio services," Millennium Conference on Antennas \& Propagation AP2000, Davos, Switzerland, April, 914, 2000, CD-ROM

[11]. K. C. Gupta et al, "Microstrip lines and Slot lines", p. 392, second edition, Artech house, 1996

[12]. "International technology roadmap for semiconductors", Semiconductor Industry Association, 2003.

[13]. L. Larson, "Silicon technology tradeoffs for radiofrequency/mixed-signal systems-on-a-chip", IEEE Transactions on Electron Devices, Volume 50, Issue 3, March 2003 Page(s): 683 - 699.

[14]. J.A. Kaiser, "The Archimedean two-wire spiral antenna," IRE Trans. on Antennas and Propagation, vol. 8, May, pp. 312-323, 1960

[15]. R. N., Simons, "Coplanar waveguide circuits, components, and systems", p.93, p. 155, John Wiley \& Sons, Inc, 2001.

[16]. J. Thaysen et al. $\mathrm{n}$ "Characterization and Optimization of a Coplanar Waveguide Fed Logarithmic Spiral Antenna" IEEE AP-S Conference on Antennas and Propagation for Wireless Communications, pp 25-28 November2000

[17]. S.A. Tretyakov et al., "Wire antennas near artificial impedance surfaces", Microwave Opt Technology Letters 27 (2000), 46-50. 